\title{
2017 Breakthrough Prizes Awarded
}

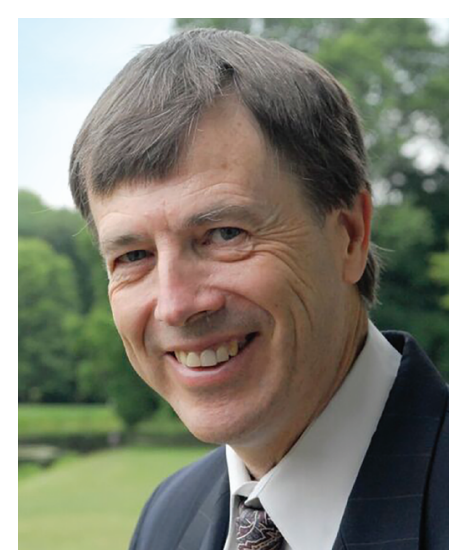

Jean Bourgain

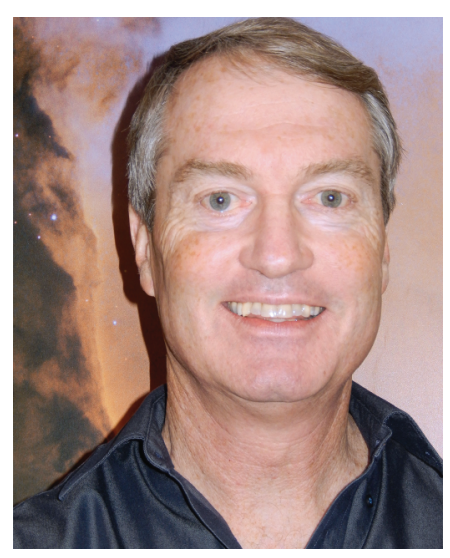

Joseph Polchinski

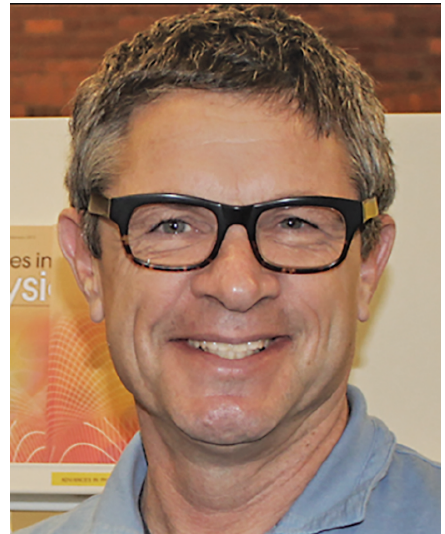

Andrew Strominger

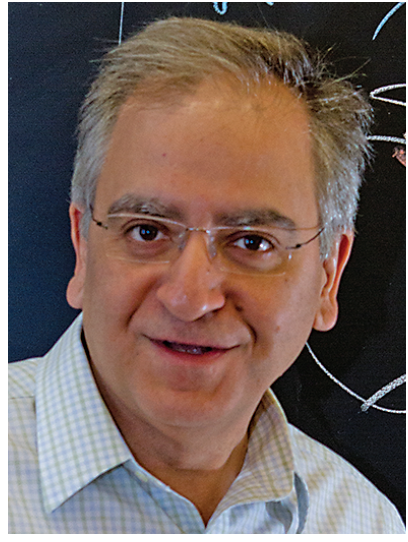

Cumrun Vafa

\section{Breakthrough Prize in Mathematics}

JEAN Bourgain of the Institute for Advanced Study, Princeton, New Jersey, has been selected as the recipient of the 2017 Breakthrough Prize in Mathematics by the Breakthrough Prize Foundation. Bourgain was honored for "major contributions across an incredibly diverse range of areas, including harmonic analysis, functional analysis, ergodic theory, partial differential equations, mathematical physics, combinatorics, and theoretical computer science." The prize carries a cash award of US\$3 million.

The Notices asked Terence Tao of the University of California Los Angeles to comment on the work of Bourgain (Tao was on the Breakthrough Prize committee and was also one of the nominators of Bourgain). Tao responded: "Jean Bourgain is an unparalleled problem-solver in analysis who has revolutionized many areas of the subject by introducing new techniques and ideas. Some of his earliest contributions include his solution of the $\Lambda$ (p) problem in Fourier analysis, his advances on the Kakeya and restriction problems in harmonic analysis and the application of related estimates to nonlinear partial differential equations, and his numerous works in high dimensional geometry, for instance in establishing the reverse Santaló inequality with Milman or the restricted invertibility theorem with Tzafriri. In the last decade, Bourgain's investigations into the sum-product phenomenon have led to deep new exponential sum estimates in number theory (which in some regimes can even improve on what can

DOI: http://dx.doi.org/10.1090/noti1509

be obtained from the results of Weil and Deligne on the Riemann hypothesis over finite fields), as well as the development (with Gamburd and Sarnak) of the affine sieve that has proven to be a powerful tool for analyzing thin groups. Most recently, with Demeter and Guth, Bourgain has established several important decoupling theorems in Fourier analysis that have had applications to partial differential equations, combinatorial incidence geometry, and analytic number theory, in particular solving the Main Conjecture of Vinogradov, as well as obtaining new bounds on the Riemann zeta function."

\section{Biographical Sketch: Jean Bourgain}

Jean Bourgain was born in 1954 in Oostende, Belgium. He received his $\mathrm{PhD}$ (1977) and his habilitation (1979) from the Free University of Brussels. He was a professor at the University of Brussels and at the University of Illinois at Urbana-Champaign, moving in 1985 to the Institut des Hautes Études Scientifiques in Paris. Ten years later, he became a professor at the Institute for Advanced Study in Princeton, where he is currently the IBM von Neumann Professor of Mathematics. In 1994, he received the Fields Medal. His other distinctions include the Salem Prize (1983), the Élie Cartan Prize of the French Academy of Sciences (1990), the Ostrowski Prize (1991), the Shaw Prize in Mathematical Sciences (2010), and the Crafoord Prize in Mathematics of the Royal Swedish Academy of Sciences (2012). 


\section{Breakthrough Prize in Fundamental Physics}

JOSEPH POLCHINSKI of the Kavli Institute for Theoretical Physics, University of California Santa Barbara, and ANDREW STROMINGER and CUMRUN VAFA, both of Harvard University, were awarded the Breakthrough Prize in Fundamental Physics for their "serious advances in string theory, the alleged but still unproven theory of everything, and what it might mean for black holes and the universe." They will share the cash award of US $\$ 3$ million.

The Notices asked Edward Witten of the Institute for Advanced Study in Princeton to comment on the work of Polchinski, Strominger, and Vafa. Witten responded: "Polchinski, Strominger, and Vafa are known for deep and far-reaching contributions to the understanding of quantum field theory and string theory, with implications for a variety of areas of physics and mathematics.

"Polchinski's single most celebrated contribution, dating back to the early to mid-1990s, has been the theory of D-branes. Certain string theories that semiclassically have closed strings only-meaning that the world history of the string is a two-manifold without boundary-turn out in a full quantum treatment to also have open strings, corresponding to world histories with boundary. D-branes provided a crucial link between string theory and gauge theory and have had dramatic consequences-developed in part in some of Polchinski's later papers-for the understanding of quantum 'dualities' among string theories and field theories. For physicists, these dualities are an essential part of understanding what happens when quantum effects are strong and a semiclassical approximation is no longer useful. Mathematically, they have many much-studied manifestations, ranging from mirror symmetry to the geometric Langlands correspondence.

"The single most celebrated contribution of Strominger and Vafa involved the quantum mechanics of black holes. In the early 1970s, Jacob Bekenstein and Stephen Hawking had interpreted the area of the horizon of a black hole as a form of entropy. This led many physicists to suspect that when the black hole is studied quantum mechanically, its horizon area would equal the logarithm of the number of its quantum states. For a long time, however, it was impossible to demonstrate this through any sort of concrete computation. At first, a suitable quantum gravity theory in which one might do the computation was not available, and later, when string theory emerged, such a calculation was technically out of reach. The first such calculation was accomplished by Strominger and Vafa in a dramatic 1996 paper. Their main technical tool was to interpret the quantum states of a black hole, in an appropriate context, in terms of the elliptic genus of the moduli space of vector bundles on a $K 3$ surface. The connection between black holes and vector bundles was deduced from the link between string theory and gauge theory that is provided by D-branes.

"Many other contributions by Strominger and Vafa have been influential in mathematics as well as physics. Only a few examples can be cited here. In the mid1980s, Strominger was one of the initiators of the use of Calabi-Yau manifolds to construct models of particle physics and made many of the important contributions in this area. One milestone was his discovery, with Brian Greene and David Morrison, of a link between singularities of a Calabi-Yau manifold and phase transitions in physics. Vafa was one of the originators of the concept of mirror symmetry between a complex manifold and a symplectic manifold and contributed many key ideas in this subject. He also introduced $F$-theory, an extension of string theory using elliptic fibrations. With Rajesh Gopakumar, Vafa introduced in 1998 what mathematically would be called a categorification of the Gromov-Witten invariants; the following year, with Hirosi Ooguri, he developed an analog of this for knot theory.

"Polchinski's other work defies easy summary. He introduced a new point of view about the renormalization group in condensed matter physics and relativistic field theory. He has made many penetrating contributions to the understanding of gauge/string duality and its implications for our understanding of gauge theories. And in recent years, initially with Ahmed Almheiri, Donald Marolf, and James Sully, he introduced and developed the baffling and highly influential 'firewall' paradox involving quantum black holes, which still today presents a deep challenge to our understanding of fundamental physics."

\section{Biographical Sketch: Joseph Polchinski}

Joseph Polchinski was born May 16, 1954, in White Plains, New York. He received his $\mathrm{PhD}$ from the University of California Berkeley in 1980 under Stanley Mandelstam. He was professor at the University of Texas at Austin (1984-1992) before joining U Cal Santa Barbara and the Kavli Institute. Among his honors are the Dannie Heineman Prize for Mathematical Physics (2007), the Dirac Medal (2008), and the Physics Frontiers Breakthrough Prize (2013). He is the author of the two-volume textbook String Theory (1998).

\section{Biographical Sketch: Andrew Strominger}

Andrew Strominger was born in 1955 and received his $\mathrm{PhD}$ in 1982 from the Massachusetts Institute of Technology under the supervision of Roman Jackiw. He held a faculty position at the University of California Santa Barbara before joining the faculty of Harvard University in 1997. His honors include the Physics Frontiers Breakthrough Prize (with Cumrun Vafa, 2012), the Klein and Dirac Medals (2014), and the Dannie Heineman Prize for Mathematical Physics (2016).

\section{Biographical Sketch: Cumrun Vafa}

Cumrun Vafa was born in Tehran, Iran, in 1960 and received his $\mathrm{PhD}$ from Princeton University under the direction of Edward Witten. His honors include the AMS Leonard Eisenbud Prize (2008), the Dirac Medal of the ICTP (2008), the Breakthrough Prize in Fundamental Physics (with Andrew Strominger, 2012), and the Dannie Heineman Prize for Mathematical Physics (2016).

\section{About the Prize}

The Breakthrough Prize in Mathematics was created by Mark Zuckerberg and Yuri Milner in 2013. It aims to rec- 


\section{COMMUNICATION}

ognize major advances in the field, to honor the world's best mathematicians, to support their future endeavors, and to communicate the excitement of mathematics to the general public. The prize is accompanied by a cash award of US\$3 million. Previous winners are Simon Donaldson, Maxim Kontsevich, Jacob Lurie, Terence Tao, Richard Taylor (2015), and Ian Agol (2016).

The Breakthrough Prize in Fundamental Physics was founded in 2012 by Yuri Milner to recognize those individuals who have made profound contributions to human knowledge. It is open to all physicists - theoretical, mathematical, experimental-working on the deepest mysteries of the Universe. The inaugural Breakthrough Prize ceremony was held March 20, 2013, in Geneva, Switzerland, where the Prize was won by Alexander Polyakov. Two Special Fundamental Physics Prizes of \$3 million were awarded to Stephen Hawking and to seven CERN scientists with leading roles in the discovery of the the Higgs boson.

-Breakthrough Prize Committee Announcement

\section{Photo Credits}

Photo of Jean Bourgain is courtesy of Cliff Moore.

Photo of Andrew Strominger is courtesy of Marina Werbeloff, Harvard University.

Photo of Joseph Polchinski is courtesy of KITP.

Photo of Cumrun Vafa is courtesy of Hayward Photography.

\section{American Mathematical Society}

\section{Let Your Voice Be Heard!}

\section{Do you enjoy what you're reading in the Notices?}

\section{Would you like to see an article on a certain topic? Person? Situation?}

Then we want to hear from you. Use our Notices Online Commentary Feature to post your general comments and suggestions:

\section{www.ams_org/notices}

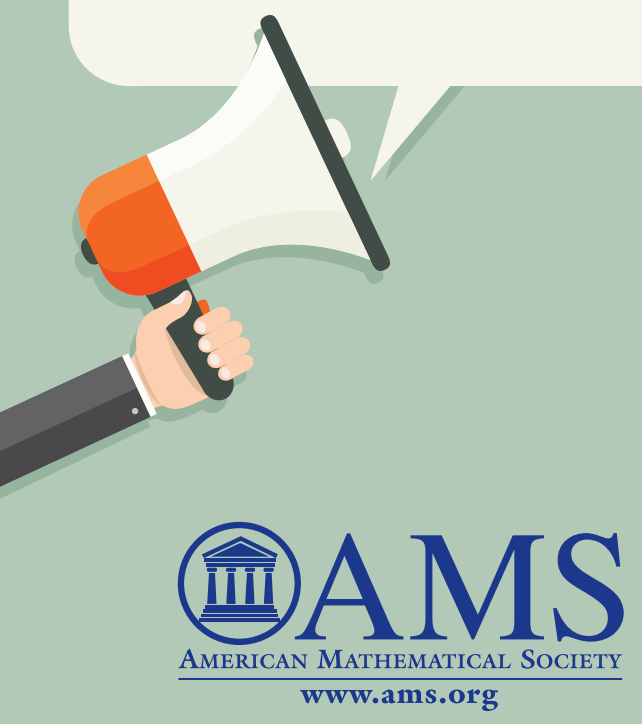

